\title{
Avaliação do Ombro em Pacientes Portadores de Espondilite Anquilosante por meio de Ultra-som
}

\author{
Evaluation of the Shoulder in Patients with \\ Ankylosing Spondylitis with Ultrasound
}

\author{
Ciro Yoshida Junior ${ }^{(1)}$, Edson Shinji Kubota(2), Themis Mizerkowski Torres ${ }^{(3)}$, \\ Rozana Mesquita Ciconelli ${ }^{(4)}$, Artur da Rocha Corrêa Fernandes ${ }^{(5)}$
}

\begin{abstract}
RESUMO
Objetivo: Descrever as principais alterações nos ombros de pacientes portadores de espondilite anquilosante (EA) por meio de ultra-som (US) e correlacionar os achados de imagem com a idade e o tempo de doença. Métodos: Foram selecionados 35 pacientes com diagnóstico clínico de EA. Os pacientes foram submetidos a exame de ultra-sonografia dos ombros, sendo avaliados os tendões do manguito rotador quanto à sua ecotextura e espessura, o tendão do bíceps, a articulação acromioclavicular, os tubérculos umerais, a bursa subacromial-subdeltóidea, e a face posterior da articulação glenoumeral. Foram colhidos dados de tempo de doença, presença de dor nos ombros e freqüiência das alterações das estruturas avaliadas. Os dados foram comparados com a idade e o tempo de doença, utilizando-se teste qui-quadrado e teste t de Student. Resultados: Foi observada prevalência de $20 \%$ de alterações no manguito rotador, sendo $10 \%$ de tendinose/calcificação tendínea e $10 \%$ de rupturas tendíneas. A espessura média dos tendões do manguito rotador foi de $5,8 \mathrm{~mm}$. As alterações ósseas nos tubérculos do úmero ocorreram em $84,3 \%$ dos ombros e as alterações acromioclaviculares em 54,3\% dos ombros. Conclusão: Não há modificação da espessura dos tendões do manguito rotador relacionadas com o tempo de doença. Os principais achados de US, que se correlacionam com o tempo de doença na EA, são alterações ósseas nos tubérculos umerais e na articulação acromioclavicular. Não houve relação entre tempo de doença e presença de lesão tendínea.
\end{abstract}

Palavras-chave: espondilite anquilosante, ultra-som, ombro.

\section{INTRODUÇÃO}

A espondilite anquilosante (EA) é uma desordem de inflamação crônica de causa desconhecida que afeta principalmente o esqueleto axial, embora o esqueleto apendicular também possa ser significativamente envolvido. A EA afeta

\begin{abstract}
Purpose: To describe shoulder involvement and its prevalence with ultrasound in patients with ankylosing spondylitis and correlate the findings with age and duration of disease. Methods: Ultrasound scans of both shoulders were performed in thirty five selected patients with clinical diagnosis of ankylosing spondylitis. Rotator cuff texture and thickness were evaluated as well as the biceps tendon, acromioclavicular joint, humeral head tuberosities, subacromial-subdeltoid bursa, and posterior glenohumeral joint. Besides the prevalence of shoulder involvement, information about presence of pain and duration of disease were registered. The data were compared with age and duration of disease using chi-square and Student's t tests. Results: The prevalence of rotator cuff disease was 20\%; with $10 \%$ of tendinosis/tendon calcification and $10 \%$ of partial/ full thickness tears. The mean rotator cuff thickness was 5.8 $\mathrm{mm}$. Irregularities of the humeral head tuberosities were present in $84.3 \%$ of shoulders and acromioclavicular irregularities were present in $54.3 \%$ of shoulders. Conclusion: There was no change in rotator cuff thickness related with duration of disease. Bony irregularities in humeral head tuberosities and acromioclavicular joint were the main finding associated with duration of disease. There was no relation between duration of disease and presence of tendinous disease.
\end{abstract}

Keywords: ankylosing spondylitis, ultrasound, shoulder.

articulações sinoviais e cartilaginosas, assim como locais de inserção tendínea e ligamentar ao osso (ênteses), com predileção por articulações do esqueleto axial, especialmente articulações sacroilíacas, apófises, discovertebral e costovertebral. Estudos descrevendo o envolvimento articular periférico na EA são poucos, porém, manifestações

Recebido em 27/6/2007. Aprovado, após revisão, em 21/3/2008. Declaramos a inexistência de conflitos de interesse

Departamento de Diagnóstico por Imagem da Universidade Federal de São Paulo/Escola Paulista de Medicina (UNIFESP/EPM).

1. Médico radiologista, especialista em Radiologia e Diagnóstico por Imagem pelo Colégio Brasileiro de Radiologia.

2. Médico radiologista, colaborador do Departamento de Diagnóstico por Imagem UNIFESP/EPM

3. Médica reumatologista, pós-graduanda da Disciplina de Reumatologia da UNIFESP/EPM.

4. Médica reumatologista, professora visitante da Disciplina de Reumatologia da UNIFESP/EPM.

5. Médico radiologista, professor adjunto do Departamento de Diagnóstico por Imagem UNIFESP/EPM

Endereço para correspondência: Ciro Yoshida Junior, Rua Francisco Barbosa Lima, 129, 04677-060, São Paulo, SP, e-mail: ciroyjr@gmail.com 
clínicas dessas articulações não são infreqüentes, tendo sido documentadas em $40 \%$ dos pacientes com EA acompanhados por dois anos ${ }^{(1)}$. O envolvimento de articulações periféricas geralmente é observado junto ou pouco depois do envolvimento de articulações centrais, podendo ainda, ocasionalmente, preceder os sintomas centrais e ser a queixa predominante ${ }^{(2)}$. Outro estudo verificou que o envolvimento de grandes articulações proximais pode chegar a até $50 \%$ dos pacientes com EA grave internados em um hospital ${ }^{(3)}$. Entre as articulações periféricas, o ombro destaca-se por ser a segunda mais acometida (após o quadril) em pacientes com EA de longa data, ocorrendo em $32 \%$ dos pacientes ${ }^{(2)}$. Verificou-se que o envolvimento de articulações periféricas é um importante fator de prognóstico da doençą ${ }^{(1,4)}$ e o envolvimento do ombro é um marcador de doença mais grave e de maior impacto na qualidade de vida do paciente ${ }^{(3)}$. Pacientes com comprometimento periférico pela doença queixam-se com mais freqüência e intensidade de dores, têm menor atividade física e têm maior prevalência de depressão e ansiedade ${ }^{(3)}$.

A maioria dos trabalhos publicados na literatura usou a radiografia como método para avaliação do ombro na EA e, raramente, foi usada a ressonância magnética ${ }^{(3,5,6)}$. Objetivamos descrever as alterações nos ombros de pacientes com EA por ultra-som (US), uma vez que não foram encontrados estudos do ombro exclusivamente por este método na literatura pesquisada.

\section{MÉTODOS}

Foram incluídos inicialmente no estudo todos os pacientes com diagnóstico clínico de EA em acompanhamento no ambulatório de artropatias soronegativas do Departamento de Reumatologia da Universidade Federal de São Paulo (UNIFESP/EPM). Foi utilizado como critério de inclusão pacientes com EA, segundo os critérios diagnósticos de Nova York modificado ${ }^{(7)}$. Foram excluídos do estudo todos os pacientes que apresentassem condições nos ombros não relacionadas à doença de base, como fratura no ombro, luxação, procedimentos cirúrgicos e infecção. Dos pacientes em acompanhamento no ambulatório foram selecionados 35 , que realizaram exame de ultra-sonografia dos ombros, no período entre julho e setembro de 2004. Esse grupo incluiu 29 homens e 6 mulheres, com média de idade de 39,6 anos (amplitude de 20 a 66 anos). A duração média da doença foi de 12,9 anos (amplitude de 2 a 40 anos). Os pacientes foram interrogados quanto à presença de dor igual ou superior a um mês de duração no ombro, uni ou bilateral.

Os exames de ultra-som foram realizados por um radiologista experiente com conhecimento prévio do diagnóstico dos pacientes. Esses foram examinados sentados, utilizando transdutor linear de 4-9 MHz (Sonoline Antares, Siemens Medical Solutions Inc., Erlangen, Alemanha). Foram avaliados os tendões do manguito rotador quanto a sua ecotextura e espessura, o tendão do bíceps, a articulação acromioclavicular, os tubérculos umerais, a bursa subacromial-subdeltóidea e a face posterior da articulação glenoumeral, utilizando-se as técnicas descritas na literatura ${ }^{(8,9)}$. Os dados obtidos foram comparados com a idade e o tempo de doença empregando-se os testes quiquadrado e $t$ de Student. O estudo obteve a aprovação do comitê de ética da instituição.

\section{RESULTADOS}

Entre os 70 ombros estudados, 28 (40\%) eram de pacientes com queixas de dor e $42(60 \%)$ sem dor. Entre os homens, observou-se $36(51,4 \%)$ ombros não dolorosos e $22(31,4 \%)$ dolorosos. Entre as mulheres, observou-se $6(8,5 \%)$ ombros não-dolorosos e $6(8,5 \%)$ dolorosos. $\mathrm{E}$ quando avaliamos a frequiência de dor nos ombros por paciente, obtivemos $51,4 \%$ (18/35 pacientes) de prevalência de dor nos ombros.

De todos os ombros estudados, 11 (15,7\%) não apresentaram nenhuma alteração do contorno ósseo dos tubérculos umerais, enquanto $59(84,3 \%)$ apresentaram algum grau de alteração do contorno ósseo (Figura 1). Dos ombros examinados, $38(54,3 \%)$ apresentaram irregularidade do contorno da articulação acromioclavicular e $32(45,7 \%)$ tinham contornos regulares (Figura 2).

Das alterações observadas na bursa subacromial-subdeltóidea, um (1,4\%) ombro tinha espessamento da bursa e oito $(11,4 \%)$ continham líquido no seu interior. Os 61 ombros restantes $(87,1 \%)$ não tinham alterações na bursa. Não encontramos nenhum ombro com derrame na articulação glenoumeral na avaliação posterior do ombro.

Em relação às alterações do tendão da cabeça longa do bíceps e sua bainha sinovial, observamos espessamento sinovial em dois $(2,9 \%)$ ombros, presença de líquido em três $(4,3 \%)$ ombros, líquido e espessamento sinovial em um $(1,4 \%)$ ombro, e $64(91,5 \%)$ ombros normais. Não foi observada nenhuma alteração de ecotextura ou morfologia nos tendões dos bíceps analisados. 

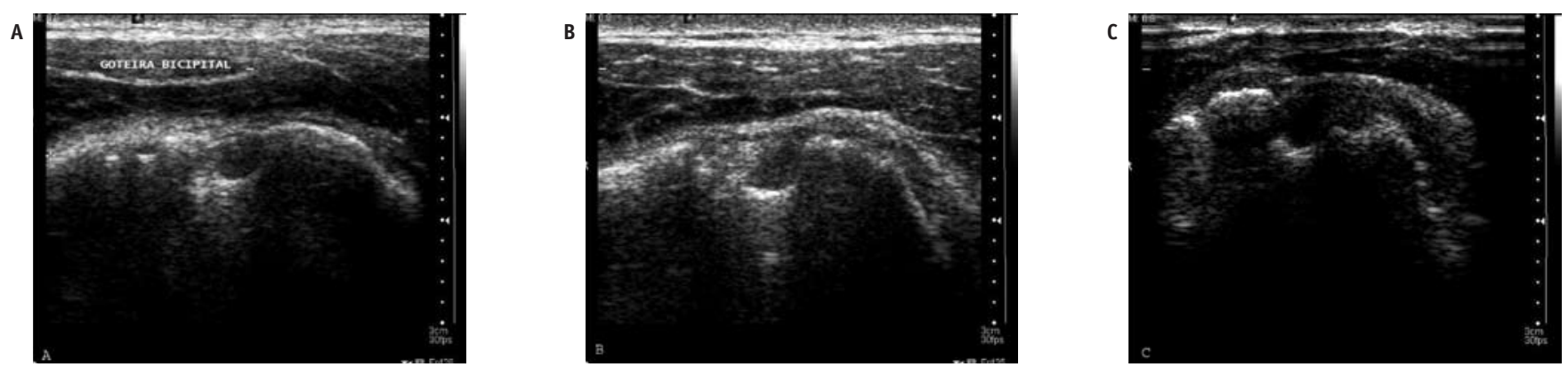

Figura 1 - Irregularidade óssea dos tubérculos junto à goteira biciptal. A. Irregularidade do contorno ósseo apenas no tubérculo maior. B. Irregularidade do contorno ósseo nos dois tubérculos. C. Irregularidade óssea acentuada em ambos os tubérculos.
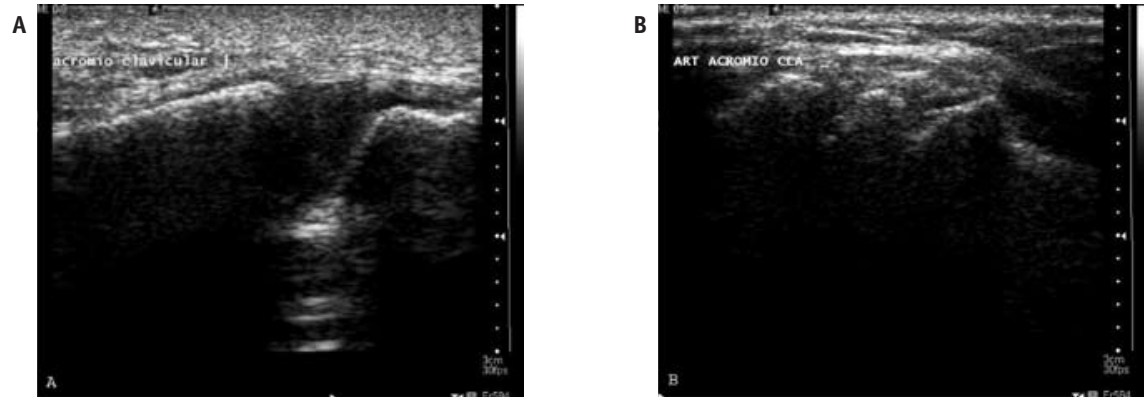

Figura 2 - Articulação acromioclavicular: achados ao US. A. Contorno ósseo regular da articulação acromioclavicular. B. Contorno ósseo acentuadamente irregular da articulação acromioclavicular.

Foi observada prevalência de $20 \%$ (14/70 ombros) de alterações no manguito rotador, sendo $10 \%(7 / 70)$ de tendinose/calcificação tendínea e 10\% (7/70) de rupturas tendíneas (Figura 3). A média de espessura para o tendão subescapular foi de 5,4 $\pm 1,1 \mathrm{~mm}$ à direita e $5,4 \pm 1,1 \mathrm{~mm}$ à esquerda. Para o tendão supra-espinal a média foi de 6,6 $\pm 1,5 \mathrm{~mm}$ à direita e $6,4 \pm 1,4 \mathrm{~mm}$ à esquerda. E para $\mathrm{o}$ tendão infra-espinal a média de espessura dos tendões foi de 5,6 $\pm 1,4 \mathrm{~mm}$ à direita e $5,5 \pm 0,9 \mathrm{~mm}$ à esquerda. No caso de ruptura completa do tendão, observada em apenas l caso, foi atribuído valor zero para espessura do tendão para fins de cálculos estatísticos. A média da espessura do manguito como um todo, obtendo-se a média dos três tendões, foi de 5,8 $\pm 1,3 \mathrm{~mm}$. Não observamos diferença estatisticamente significativa na média de espessura dos tendões quando agrupamos e comparamos um grupo de pacientes com sete anos ou mais de doença e outro grupo com menos de sete anos de doença $(\mathrm{p}=0,23$ para tendão subescapular; $\mathrm{p}=0,37$ para tendão supra-espinal; $\mathrm{p}=0,77$ para tendão infra-espinal) (Tabela 1 ).
Ainda separando os pacientes nos dois grupos, um com sete anos ou mais de doença, e outro com menos de sete anos, observamos maior prevalência de irregularidade do contorno ósseo da articulação acromioclavicular e dos tubérculos umerais (66\% e $94 \%$, respectivamente) no primeiro grupo em comparação com o de menos tempo de doença $(25 \%$ e $60 \%$, respectivamente) $(\mathrm{p}=0,003$ e $\mathrm{p}=0,001$, respectivamente) (Tabela 1 ). Em relação a presença ou ausência de alterações na bursa subacromial-subdeltóidea e dor nos ombros, observamos maior prevalência de eventos positivos no grupo com mais tempo de doença (18\% e 46\%, respectivamente) em relação ao segundo grupo ( $0 \%$ e $25 \%$, respectivamente), com tendência a atingir nível de significância estatística $(\mathrm{p}=0,052$ e $\mathrm{p}=0,058$, respectivamente) (Tabela 2). E, finalmente, quando analisamos os dois grupos quanto à presença ou à ausência de alterações no manguito rotador (tendões subescapular, supra-espinal e infra-espinal), observamos maior prevalência no primeiro grupo $(10 \%, 16 \%$, $1,4 \%$, respectivamente), contra $5 \%, 15 \%$ e $0 \%$, para o segundo grupo $(\mathrm{p}=0,6, \mathrm{p}=1, \mathrm{p}=1$, respectivamente) (Tabela 3$)$. 

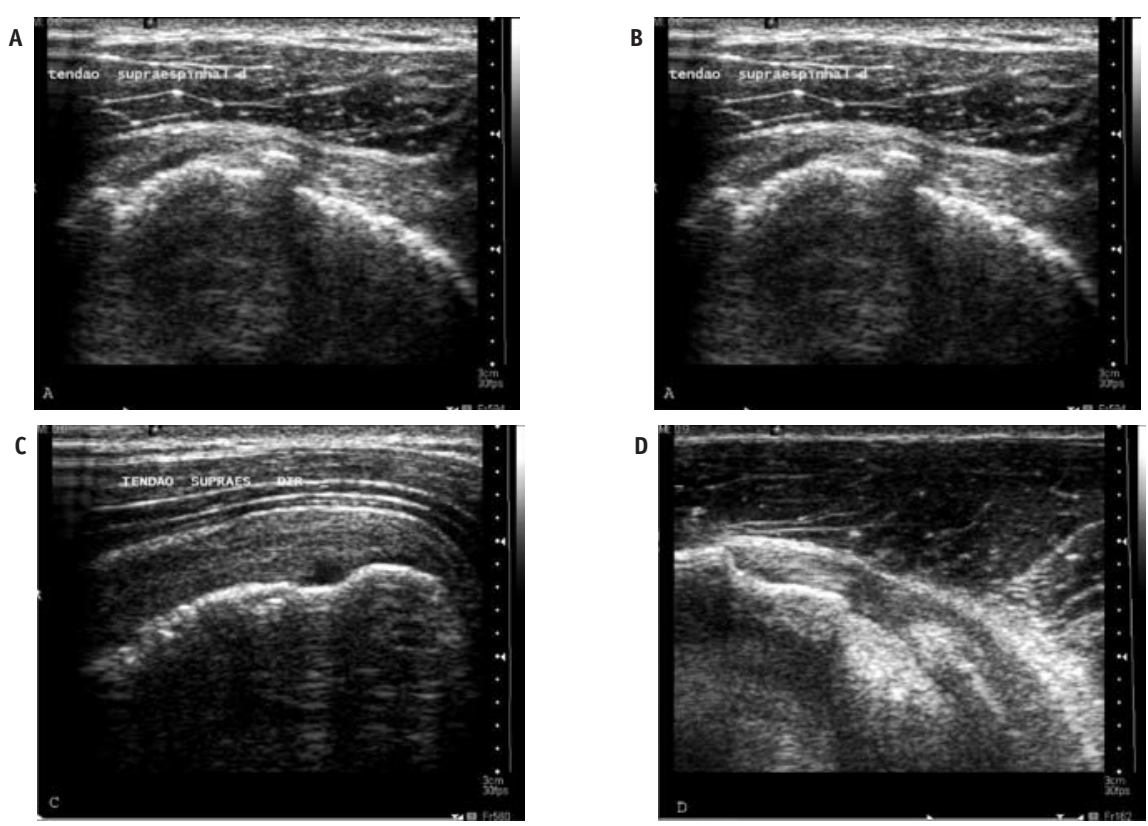

Figura 3 - Alterações tendíneas observadas ao US. A. Calcificação tendínea do supra-espinal, que se apresenta heterogênea. Nota-se ainda irregularidade óssea do tubérculo maior. B. Espessamento e heterogeneidade (tendinose) do tendão do supra-espinal. C. Presença de área focal de hipoecogenicidade do tendão supra-espinal na superfície articular, caracterizando ruptura parcial. D. Afilamento com descontinuidade tendínea e concavidade da face bursal, caracterizando ruptura completa.

TABela 1

Comparação ENTre tempo de DOENÇa E VARIÁveis observadas

\begin{tabular}{|c|c|c|c|c|c|c|c|c|}
\hline \multirow[t]{3}{*}{ Tempo de doença } & \multirow[t]{3}{*}{$\mathrm{N}$} & \multicolumn{7}{|c|}{ Variáveis } \\
\hline & & \multicolumn{2}{|c|}{$\begin{array}{l}\text { Irregularidade óssea } \\
\text { nos tubérculos }\end{array}$} & \multicolumn{2}{|c|}{$\begin{array}{l}\text { Irregularidade óssea na } \\
\text { articulação acromioclavicular }\end{array}$} & \multicolumn{3}{|c|}{ Média ( \pm desvio-padrão) da espessura do tendão ( $\mathrm{mm}$} \\
\hline & & Não & Sim & Não & Sim & Subescapular & Supra-espinal & Infra-espinal \\
\hline$<7$ anos & 20 & 8 & 12 & 15 & 5 & $5,1( \pm 1,0)$ & $6,8( \pm 0,8)$ & $5,5( \pm 1,1)$ \\
\hline$\geq 7$ anos & 50 & 3 & 47 & 17 & 33 & $5,5( \pm 1,1)$ & $6,4( \pm 1,6)$ & $5,6( \pm 1,2)$ \\
\hline Total & 70 & 11 & 59 & 32 & 38 & $5,4( \pm 1,1)$ & $6,5( \pm 1,4)$ & $5,5( \pm 1,2)$ \\
\hline Valor de $p$ & & \multicolumn{2}{|c|}{$(p=0,001)$} & \multicolumn{2}{|c|}{$(p=0,003)$} & $(p=0,23)$ & $(p=0,37)$ & $(p=0,77)$ \\
\hline
\end{tabular}

TABela 2

COMPARAÇÃo ENTRE TEMPO DE DOENCA E VARIÁVEIS OBSERVADAS

\begin{tabular}{lccccc}
\hline Tempo de doença & \multicolumn{5}{c}{ Variáveis } \\
\hline & Dor nos ombros & $\begin{array}{c}\text { Alteração na bursa } \\
\text { subacromial-subdeltóidea }\end{array}$ & Total \\
\hline & Não & Sim & Não & Sim & \\
\hline$<7$ anos & 15 & 5 & 20 & 0 & 20 \\
$\geq 7$ anos & 27 & 23 & 41 & 9 & 50 \\
Total & 42 & 28 & 61 & 9 & 70 \\
$X^{2}=$ & $3,9(p=0,058)$ & $4,1(p=0,052)$ & \\
\hline
\end{tabular}

\section{DISCUSSÃO}

Existem poucos estudos de imagem do ombro na EA publicados na literatura. Embora as alterações dos ombros na EA não possam ser consideradas importantes no critério
TABELA 3

COMPARACÃo ENTRE TEMPO DE DOENÇA E ALTERAÇÕES TENDÍNEAS

\begin{tabular}{|c|c|c|c|c|c|c|c|}
\hline \multirow[t]{3}{*}{ Tempo de doença } & \multicolumn{7}{|c|}{ Alterações tendíneas } \\
\hline & \multicolumn{2}{|c|}{ Subescapular } & \multicolumn{2}{|c|}{ Supra-espinal } & \multicolumn{2}{|c|}{ Infra-espinal } & \multirow[t]{2}{*}{ Total } \\
\hline & Não & Sim & Não & Sim & Não & Sim & \\
\hline$<7$ anos & 19 & 1 & 17 & 3 & 20 & 0 & 20 \\
\hline$\geq 7$ anos & 45 & 5 & 42 & 8 & 49 & 1 & 50 \\
\hline Total & 64 & 6 & 59 & 11 & 69 & 1 & 70 \\
\hline$x^{2}=$ & \multicolumn{2}{|c|}{$0,5(p=0,6)$} & \multicolumn{2}{|c|}{$0,1(p=1)$} & \multicolumn{2}{|c|}{$0,4(p=1)$} & \\
\hline
\end{tabular}

diagnóstico, elas podem fornecer pistas adicionais para o diagnóstico da doença. Esses poucos estudos realizados usaram a radiografia como método de imagem para o estudo do ombro $^{(3,6)}$. Não encontramos estudo do ombro por meio de US na EA na literatura pesquisada. 
Irregularidades do contorno ósseo foram as principais alterações observadas no US, ocorrendo em $84,3 \%$ (59/70) dos ombros para os tubérculos em 54,3\% (38/70) para a articulação acromioclavicular, e estavam associadas com o tempo de doença.

O envolvimento inflamatório de articulações sinoviais é comum em pacientes com espondiloartropatias, e está associado com osteíte difusa do osso subjacente à inserção capsular ${ }^{(10)}$. As alterações na articulação acromioclavicular descritas na literatura foram baseadas em radiografias e a presença de erosões, proliferação óssea e entesopatia confirmaram nossos achados ${ }^{(3,6,11)}$. Will et al. ${ }^{(3)}$ observaram erosões e proliferação óssea/entesopatia da articulação acromioclavicular em, respectivamente, $17,3 \%$ e 19,2\% de 52 ombros de pacientes com EA nas radiografias. Um recente estudo por meio de ressonância magnética (RM) em 17 ombros de pacientes com EA observou que a artrose da articulação acromioclavicular foi o achado mais prevalente, constatado em $94,1 \%$ dos ombros, contra $68,1 \%$ no grupocontrole. No entanto, todos os pacientes com EA tinham evidência clínica de alteração do ombro ${ }^{(5)}$.

As alterações ósseas relacionadas ao manguito rotador também foram observadas por van Holsbeeck et al. ${ }^{(12)}$. Estes autores relataram irregularidades no tubérculo maior de pacientes com ruptura tendínea parcial no US e concluíram que as irregularidades podem ajudar a confirmar o diagnóstico de ruptura parcial em casos duvidosos. Em nosso estudo, houve concordância de alterações ósseas com lesões tendíneas em 13 ombros, refletindo que na nossa amostra houve ocorrência maior de irregularidade óssea, independentemente de ocorrer lesão tendínea, e que as irregularidades ósseas devam surgir primeiro na doença.

Estudo de Wohlwend et al. ${ }^{(13)}$, avaliando 154 ombros assintomáticos por US, encontraram irregularidade óssea no tubérculo maior em $90 \%$ dos ombros com ruptura do manguito rotador, inferindo forte associação entre irregularidade do tubérculo e lesão tendínea. Encontramos irregularidade óssea nos tubérculos em 100\% (7/7 ombros) dos ombros com ruptura tendínea do manguito rotador, confirmando a hipótese do estudo antes descrito. Entretanto, encontramos muitos ombros com irregularidade óssea mas sem lesão tendínea; em 82,5\% (52/63 ombros) dos ombros. Wohlwend et al. ${ }^{(13)}$ encontraram apenas $11 \%$ de irregularidade nos tubérculos nos ombros sem lesão tendínea. Portanto, deve haver algum mecanismo fisiopatológico adicional que favoreça a formação dessas irregularidades nos tubérculos de pacientes com EA.
Foi observada correlação estatisticamente significativa entre tempo de doença e presença de irregularidade óssea $(\mathrm{p}=0,001)$ (Tabela 1$)$. Outra possível hipótese para as alterações ósseas poderia estar relacionada à doença de base. Em 2001, Benjamin e McGonagle ${ }^{(14)}$, discutindo a base anatômica para a localização da doença nas ênteses nas espondiloartropatias, citam que a EA acomete a interface entre o osso e a cartilagem, além disso, a região do manguito rotador é local comum de microtrauma, incluindo a possibilidade de microfraturas. $\mathrm{O}$ desgaste e a ruptura tendínea estariam relacionados a estes microtraumas e reparos na fibrocartilagem da êntese. As irregularidades podem também relacionar-se à formação de esporões ósseos (entesófitos) por alterações inflamatórias difusas no osso subjacente à inserção tendínea (entesite), que está presente em cerca de $40 \%$ a $50 \%$ das espondiloartropatias ${ }^{(14)}$. Acredita-se que o mecanismo de formação dos entesófitos possa começar por invasão vascular que se estende ao longo das colunas de células fibrocartilaginosas da êntese, até mesmo na ausência de microrrupturas ou inflamação. Os capilares invadem a êntese a partir da medula óssea subjacente e criam túneis que se tornam preenchidos por $\mathrm{osso}^{(14)}$. A expressão de mediadores inflamatórios interleucina- $1 B$, catepsina $D$ e matriz metaloproteinase 1 estariam relacionadas à erosão do osso subcondral e conseqüente ruptura tendínea ${ }^{(15)}$. Portanto, essas alterações poderiam ocorrer de modo mais precoce do que as lesões tendíneas e relacionadas à doença de base (EA).

Foram observadas alterações tendíneas em 14 (20\%) dos 70 ombros, sendo 7 (10\%) de rupturas tendíneas parcial/completa e $7(10 \%)$ de tendinose/calcificação. Comparando-se a média do tempo de doença nos ombros com tendinose/calcificação tendínea com a média do tempo de doença nos paciente sem alteração tendínea, observamos não haver diferença estatisticamente significante. De maneira semelhante, comparamos a média do tempo de doença dos ombros com ruptura parcial/completa e sem ruptura, e a média do tempo de doença dos ombros com alguma alteração tendínea e sem alteração, sem obter significância estatística. Esse resultado reflete a baixa ocorrência de lesão tendínea, mesmo nos indivíduos com tempo de doença prolongado. Isso é confirmado pelo resultado do qui-quadrado, quando avaliamos a relação entre tempo de doença e presença ou ausência de lesão tendínea, na qual não foi observada associação estatisticamente significante.

Não houve diferença estatisticamente significativa entre a média de espessura dos tendões do manguito rotador nos grupos com menos de sete anos de tempo de doença e igual ou maior a sete anos (Tabela 1). Uma hipótese 
para isso seria em virtude do baixo número de ocorrência de eventos tendíneos (20\%) em nossa amostra ou, ainda, porque o comprometimento tendíneo talvez não seja tão expressivo na faixa etária avaliada.

\section{CONCLUSÕES}

As principais alterações observadas por meio do US nos ombros de pacientes com EA foram irregularidades nos tubérculos do úmero e na articulação acromioclavicular,

\section{REFERÊNCIAS}

1. Gran JT, Skomsvoll JF: The outcome of ankylosing spondylitis: a study of 100 patients. Br J Rheumatol 36(7): 766-71, 1997.

2. Resnick D: Patterns of peripheral joint disease in ankylosing spondylitis. Radiology 110(3): 523-32, 1974.

3. Will R, Kennedy G, Elswood J, et al.: Ankylosing spondylitis and the shoulder: commonly involved but infrequently disabling. J Rheumatol 27(1): 177-82, 2000.

4. Guillemin F, Briancon S, Pourel J, Gaucher A: Long-term disability and prolonged sick leaves as outcome measurements in ankylosing spondylitis. Possible predictive factors. Arthritis Rheum 33(7): 1001-6, 1990.

5. Lambert RG, Dhillon SS, Jhangri GS, et al.: High prevalence of symptomatic enthesopathy of the shoulder in ankylosing spondylitis: deltoid origin involvement constitutes a hallmark of disease. Arthritis Rheum 51(5): 681-90, 2004.

6. Emery RJ, Ho EK, Leong JC: The shoulder girdle in ankylosing spondylitis. J Bone Joint Surg Am 73(10): 1526-31, 1991.

7. van der Linden S, Valkenburg HA, Cats A: Evaluation of diagnostic criteria for ankylosing spondylitis. A proposal for modification of the New York criteria. Arthritis Rheum 27(4): 361-8, 1984.

8. Lin J, Jacobson JA, Fessell DP, Weadock WJ, Hayes CW: An illustrated tutorial of musculoskeletal sonography: part 2, upper extremity. AJR Am J Roentgenol 175(4): 1071-9, 2000. e tais achados estão nitidamente correlacionados com o tempo de doença. Não houve modificação da espessura dos tendões do manguito rotador relacionadas com o tempo de doença. Não houve correlação entre o tempo de doença e a presença ou a ausência de lesão tendínea.

\section{AGRADECIMENTOS}

À Professora Dra. Ana Luisa Godoy Fernandes, pela preciosa colaboração no tratamento dos dados estatísticos.

9. Teefey SA, Middleton WD, Yamaguchi K: Shoulder sonography. State of the art. Radiol Clin North Am 37(4): 767-85, ix, 1999.

10. McGonagle D, Gibbon W, O'Connor P, Green M, Pease C, Emery P: Characteristic magnetic resonance imaging entheseal changes of knee synovitis in spondylarthropathy. Arthritis Rheum 41(4): 694-700, 1998.

11. Resnick D: Diagnosis of bone and joint disorders. 4.ed. Philadelphia: WB Saunders; 2002.

12. van Holsbeeck MT, Kolowich PA, Eyler WR, et al.: US depiction of partial-thickness tear of the rotator cuff. Radiology 197(2): 443-6, 1995.

13. Wohlwend JR, van Holsbeeck M, Craig J, et al.: The association between irregular greater tuberosities and rotator cuff tears: a sonographic study. AJR Am J Roentgenol 171(1): 229-33, 1998.

14. Benjamin $\mathrm{M}$, McGonagle D: The anatomical basis for disease localisation in seronegative spondyloarthropathy at entheses and related sites. J Anat 199(Pt 5): 503-26, 2001.

15. Gotoh M, Hamada K, Yamakawa H, Tomonaga A, Inoue A, Fukuda H: Significance of granulation tissue in torn supraspinatus insertions: an immunohistochemical study with antibodies against interleukin-1 beta, cathepsin D, and matrix metalloprotease-1. J Orthop Res 15(1): 33-9, 1997. 